This is the post print version of the article, which has been published in Cyberpsychology, Behavior, and Social Networking. 2018, 21(2): 129-137. https://doi.org/10.1089/

cyber.2016.0728.

Kaakinen Markus, Keipi Teo, Räsänen Pekka, and Oksanen Atte

\title{
Cybercrime victimization and subjective well-being: An examination of the buffering effect hypothesis among adolescents and young adults
}

\begin{abstract}
The wealth of beneficial tools for online interaction, consumption and access to others also bring new risks for harmful experiences online. This study examines the association between cybercrime victimization and subjective well-being (SWB) and, based on the buffering effect hypothesis, tests the assumption of the protective function of social belonging in cybercrime victimization. Crossnational data from the US, UK, Germany and Finland $(N=3,557$; Internet users aged 15-30 years; $49.85 \%$ female) was analyzed using descriptive statistics and main and moderation effects models. Results show that cybercrime victimization has a negative association with SWB after adjusting for a number of confounding factors. This association concerns both general cybercrime victimization as well as sub-categories such as victimization to offensive cybercrime and cyberfraud. In line with the buffering effect hypothesis, social belonging to offline groups was shown to moderate the negative association between SWB and cybercrime victimization. The same effect was not found in the social belonging to online groups. Overall, the study indicates that, analogously to crime victimization in the offline context, cybercrime is a harmful experience whose negative effects mainly concern those users who have weak social ties offline to aid in coping with such stressors.
\end{abstract}

Keywords: cybercrime victimization, subjective well-being, social belongingness, buffering effect

\section{Introduction}

The growth of the Internet into an integral part of everyday life has brought with it a wide array of potential risk for its users. Even though the immense development in the convenience of interaction, consumption and access to others has brought vast benefits, these have also worked together to lower the cost of entry into potential criminal activities online. As such, various forms of cybercrime have become prevalent in the online space where new conditions facilitate damaging behaviors.

Cybercrime, as a term, is a broad concept that encompasses all manner of criminal activity carried out online; from institutions and companies, to collective ideological groups and individuals, various levels of society are affected by the implications of crime online ${ }^{1-5}$. Here, cybercrime refers to any criminal activity that takes place through computers or devices that function through online networks and platforms. The phenomenon can be either broad or specific, from institutional level professional hacking attacks to individual level victimization experiences. The focus here is on the latter, namely on targeted victimization on the individual level through the experiences of young Internet users in a cross-national context. 
Notably, there exists overlap in terms of what constitutes a crime online when it comes to harassment, bullying and victimization ${ }^{6}$. In this study, focus is placed specifically on negative online experiences that respondents have perceived as a crime rather than on distinguishing criminality objectively. Past research from the individual perspective has shown some of the most common forms of cybercrime as harassment, identity theft, defamation and fraud ${ }^{4-8}$. Overall, the rates of cybercrime victimization tend to be low, yet the rates for young Internet users are higher than those of older age groups. Notably, young people tend to be the most active users of the Internet and as such their victimization experiences can shed light on risk phenomena relevant to all age groups.

Past research has shown various links between crime victimization experiences and diminished well-being. In general, crime victimization has adverse psychological impacts on victims, but the consequences differ in the case of violent (e.g. assault or rape) and non-violent (e.g. property related) crimes $^{9,10}$. In a longitudinal study conducted by Mahuteau and $\mathrm{Zhu}^{11}$, victimization to both physical and property crime was found to associate with subjective well-being (SWB), which is a key positive indicator of psychological health made up of subjective cognitive and affective evaluations of one's life ${ }^{12-16}$. However, the adverse effect of victimization experience on SWB was stronger for physical violence than in the case of property crimes ${ }^{11}$.

There is an ongoing discussion over whether the distinctive features of cybercrime, such as the spatial and temporal disconnect between the offender and victim, allows for its comparison to offline crime in terms of crime occurrence and victimization ${ }^{17,18}$. However, analogously to offline crime, previous studies have shown an association between online victimization and well-being problems including depression, lower self-esteem and a decrease in the sense of coherence ${ }^{19,20}$.

Furthermore, as in the case of offline crime, victimization to offensive cybercrime may have a stronger negative association with wellbeing than property-related cybercrime. The present study analyzes the association between SWB and victimization to cybercrime in general, victimization to offensive crime carried out on third parties online (i.e. defamation, illegal threat and sexual harassment), and victimization through cyberfraud (i.e. online fraud and identity theft) while also taking into consideration possible effects from users' social circles.

The benefits of social belonging have been widely discussed in both empirical and theoretical literature ${ }^{21-23}$. The central idea of the so called buffering effect hypothesis is that social relations can foster well-being directly (as a main effect), or indirectly by emotional, informational, instrumental support or social belonging that can buffer the harmful effects of different stressful life events (as a buffering effect) ${ }^{24}$. Past work on the buffering hypothesis has shown clear indications that social relationships can indeed function in a protective manner against stressors that threaten mental health and well-being ${ }^{22}$ including crime victimization ${ }^{25-27}$.

A relevant question to ask, however, is whether the online and offline environments function equivalently in terms of the buffering hypothesis. Cole and colleagues reported that both offline and online social support protected college students against the adverse effects of peer victimization ${ }^{28}$. In studies by Minkkinen and Turja and colleagues, social belonging to primary offline groups was found to protect young people against potential negative impacts of online behavior, but a similar buffering effect was not found in the social belonging to online groups ${ }^{29,30}$. In addition, Tennant and colleagues reported no moderating role of social support gained from family, significant adults, peers and close friends in a study concerning the association of cybervictimization and depression among college students ${ }^{20}$. 
These findings highlight the complex nature of the victimization environment in addition to how social support affects victims. Given past application and findings in the online setting, the buffering hypothesis continues to be relevant in the case of online stressors, especially in terms of social support originating from offline sources.

In social psychology, the main divergence between offline and online interaction has often been attributed to the communication bandwidth that does not allow for the social presence and richness of face-to-face interaction to be transferred to computer-mediated communication $(\mathrm{CMC})^{31}$. This difference in interactional dynamics can lead to lessened intimacy and quality of communication online. However, the process of relating oneself to social networks may not be solely dependent on the amount of information transferred via the communication medium ${ }^{32}$. Even though CMC may not be equivalent to face-to-face interaction, online communities have become an increasingly important source of social identification, support, and belonging for young people especially ${ }^{33,34}$.

According to Walther's (1992) social information processing theory, online social relations can eventually extend to the same level of intimacy as offline social relations through a relatively longer relational time frame through $\mathrm{CMC}^{35}$. In addition to time, personal orientation towards online interaction can determine the relational depth of online social ties ${ }^{36}$. In line with this, online social ties tend to be of more value for those with weak ties offline ${ }^{28}$, illustrating that individual social preference is key in determining the level of relational intimacy in the online setting.

It has also been suggested that the key difference between offline and online social ties lies in the ways in which people relate to one another in offline and online networks ${ }^{37}$. Online, network management and maintenance of strategic or instrumental relationships is made convenient and the level of relational intimacy or social tie strength may not be of primary importance in motivating social interactions ${ }^{37}$. Indeed, online social ties tend to have a smaller role in young peoples' lives when compared to those formed offline ${ }^{38}$.

Drawing on previous research literature, we examine the association between cybercrime and users' mental health in this study. Our cross-national sample is collected from four technologically advanced countries with high general living standards and are thus comparable in terms of cybercrime $^{39}$, namely the United States, Finland, Germany, and the United Kingdom. We summarize with the following hypotheses:

H1) Cybercrime victimization has a negative association with subjective well being.

$\mathrm{H} 2 \mathrm{a})$ Social belongingness to offline primary groups buffers the negative association between cybercrime and SWB.

$\mathrm{H} 2 \mathrm{~b})$ Social belongingness to an online community buffers the negative association between cybercrime and SWB.

\section{Methods}

\section{Participants}

In the analysis, we use four comparable datasets of youth and young adults in the United States, Finland, Germany, and the United Kingdom. The data were collected as part of a research project designed to investigate both exposure and victimization to cyberhate among adolescents and young adults aged 15 to 30 . In the online questionnaire, respondents were asked about their psychosocial and sociodemographic characteristics, online activity and social relations in both offline and online 
contexts. The data were collected from the United States $(n=1,033)$ and Finland $(n=555)$ in spring 2013 and from the United Kingdom $(n=999)$ and Germany $(n=978)$ in spring 2014.

Demographically balanced panels were used in respondent recruitment and, in accordance with informed-consent principles, the respondents were informed about the background and purpose of the study before they were asked to voluntarily participate in research. Participants were also informed about their right to withdraw from the research once it had begun and were provided with contact information for possible further questions concerning the research project. The survey data was anonymous and no identifying information was collected from the respondents. Participants $(N$ $=3557)$ were aged 15 to $30\left(M_{\mathrm{US}}=23.09, S D_{\mathrm{US}}=4.04 ; M_{\mathrm{FIN}}=22.59, S D_{\mathrm{FIN}}=4.21 ; M_{\mathrm{GER}}=23.21\right.$, $\left.S D_{\mathrm{GER}}=3.97 ; M_{\mathrm{UK}}=23.18, S D_{\mathrm{UK}}=4.14\right)$ and were female $(50.34 \%$ US, $50.00 \%$ FIN $; 50.26 \%$ GER; $49.09 \%$ UK).

\section{Measures}

Dependent variable. $S W B$ was measured with a composite variable based on the following survey items: 1) "All things considered, how happy would you say you are?" and 2) "All things considered, how satisfied are you with your life as a whole nowadays?". For both items, the measurement scale was from 1 (extremely unhappy or extremely unsatisfied) to 10 (extremely happy or extremely satisfied). Both of these items are validated and widely used measures on $\mathrm{SWB}^{12-16}$. Responses to these questions were then combined into a sum variable with a scale of 220 and an internal consistency of 0.88 (Cronbach's alpha).

Independent variables. Victimization to cybercrime, victimization to offensive cybercrime, and victimization to cyberfraud were measured by dichotomous variables $(0=$ no victimization experience during the past three years, $1=$ at least one victimization experience during the past three years). The variable measuring victimization to cybercrime in general was based on a survey item in which the respondents were asked whether they had been subjected to cybercrime during the past three years. Those respondents answering yes to this question were then also asked whether the crime had been defamation, illegal threat, identity theft, fraud, sexual harassment, or something else. Two victimization variables were then coded on the basis of these answers. Victimization to offensive cybercrime was coded as 1 if the respondent had experiences of defamation, threat, or sexual harassment during the past three years. Victimization to cyberfraud was coded as 1 if the responded had experienced identity theft or online fraud during the past three years. Both variables were coded as 0 for respondents with no victimization experiences.

Social belonging. Social belonging in offline and online contexts was measured by variables based on survey items in which respondents were asked to indicate, on a scale from 1 to 5 , how strongly they felt belonging to their family, circle of friends, school or work community, and online community. As such, the first three variables measured participants' social belonging to primary groups that originate in the offline setting while the last measures focus on social belonging to community that originates in, yet is not necessarily restricted to, the online environment. The variable measuring social belonging in the offline context was formed by combining answers to the first three questions into a sum variable with a scale of 3-15 and a sufficient internal consistency of 0.69 (Cronbach's alpha). The variable measuring social belonging online was based on the question concerning participants' sense of belonging with an online community.

Covariates. Country information and respondents' gender, age, and the scope of online activity were used as a covariate in our analyses. The variable measuring the scope of online activity was 
counted on the basis of a survey item in which respondents were asked to indicate which online services they had been using during the past three months from a list of the 21 of the most popular online services at the time (e.g. Facebook, YouTube and Twitter). The variable adding together the total number of used services had a scale from 0-19. For further details about our analytical variables, see Table 1.

\section{Statistical techniques}

We present both descriptive statistics of our analytic variables (means and standard deviations for scaled variables and as absolute and relative proportions for categorical variables) and predictive analysis. In the predictive analysis, the association between cybercrime victimization and SWB was assessed by utilizing hierarchical linear regression models. The first model included SWB as a dependent variable and our covariates as predictors. In the second model (main effect model), cybercrime victimization was added as a predictor. The potential moderating role of social belonging, in turn, was analyzed with a third model including interaction terms between cybercrime victimization and social belonging (moderation effect model). Models 2 and 3 were estimated separately for each type of cybercrime victimization (cybercrime in general, offensive cybercrime and cyberfraud).

For each model, we report regression coefficients, standard deviations, beta coefficients $(ß)$ and statistical significance as well as coefficients of effect sizes (partial eta-squared) for significant variables and model determination (adjusted R-squared). To elaborate on the hypothesized moderation effect, we counted predictive margins of cybercrime victimization for low (one standard deviation below the mean), average (the mean), and high (one standard deviation above the mean) social belonging keeping other covariates in their mean values. Based on these estimates, we present adjusted prediction plots to visualize how the association between cybercrime victimization and SWB was moderated by social belonging. Finally, the t-test was used to assess whether the slopes for low social belonging and for average and high belonging were significantly different.

\section{Results}

In the overall sample, 6.4 percent of all respondents reported cybercrime victimization and the prevalence was relatively stable across our country samples (Table 1). Victimization to offensive cybercrime was more prevalent in the overall sample, (4.3 per cent) than victimization to cyberfraud (2.9 percent). Of crimes classified as offensive, the victimization to defamation was most prevalent ( 2.6 per cent) followed by illegal threat ( 2.2 per cent) and sexual harassment (1.1 per cent). In the case of the cyberfraud category, respondents reported being exposed to fraud online more often (1.8 per cent) than to identity thefts ( 1.5 per cent).

According to the first model of our hierarchical regression analysis (Tables 2-4), the social belonging to offline primary groups was the strongest predictor of $\mathrm{SWB}(\mathrm{B}=0.75, p<0.001)$ among our covariates as it accounted for 22 percent on the SWB's variance (partial eta-squared coefficients in Tables 2-4). Our second set of models show that victimization to cybercrime in general (Table 2) offensive cybercrime (Table 3 ) and cyberfraud (Table 4) all had negative association to SWB even after adjusting for our control variables. Persons who had experienced cybercrime victimization in general during the past three years reported, on average, one unit lower SWB $(\mathrm{B}=0.97, p<0.001)$. SWB was 1.5 units lower for those with offensive cybercrime experience $(\mathrm{B}=-1.54, p<0.000)$ and 0.7 units lower for victims of cyberfraud $(\mathrm{B}=-0.69, p<0.05)$. However, victimization to cybercrime 
in general and to offensive cybercrime and cyberfraud only accounted for approximately one percent or less of SWB's variance.

As can be seen from our third set of regression models, after adding interaction terms between cybercrime victimization and social belonging variables in the models, the interaction between cybercrime victimization and social belonging to offline primary groups was significantly associated with SWB in the case of all cybercrime victimization experiences. Adjusted predictions (Figures 1-3) show that the negative association between victimization and SWB mainly concerns those respondents with low offline belonging and the connection becomes weaker as the value of the offline belonging increases. In the case of cyberfraud (Figure 3), the predicted SWB was slightly higher for victims with high social belonging than it was for nonvictims with the same level of belonging. This difference, however, was not statistically significant as it lies within predictions' 95 percent confidence interval.

According to the t-test, the slope for low offline belonging differed significantly from the average and high belonging slopes in case of victimization to cybercrime in general ( $p$-values of 0.031 and $<0.001$ ) and cyberfraud ( $p$-values of 0.009 and $<0.001$ ) and from the high belonging slope in case of offensive cybercrime $(p=0.001)$. The interaction terms between online social belonging and victimization to cybercrime in general, offensive cybercrime and cyberfraud were not significantly associated with SWB (p-values of $0.666,0.098$ and 0.302 respectively).

\section{Discussion}

This study is the first to analyze the association between cybercrime victimization and SWB. According to our findings, victimization to cybercrime in general and to subtypes of offensive cybercrime and cyberfraud in the past three years predicted lower SWB even after adjusting for other factors including social belonging, country, gender, age and the scope of online activity. Victimization to offensive crime had a stronger negative association with SWB than cyberfraud victimization. These results are in line with earlier research on crime victimization and SWB in the offline context ${ }^{11}$. Results also reinforce past findings on the relationship between online victimization and well-being problems ${ }^{19,20}$. Thus, even though cybercrime victimization does not share the spatial and temporal connection between victims and offenders as is typical in offline crime $^{17,18}$, it has the potential to be harmful for their victims' well-being. However, victimization experiences only accounted for a small proportion of SWB's variance.

Furthermore, our hypothesis that the negative association between cybercrime victimization and SWB will be buffered by social belonging was partly supported by our data. Our findings are in line with the buffering hypothesis, one of the dominant theoretical models of social support research ${ }^{22-24}$ and they reinforce past research concerning the buffering effect in case of offline crime victimization $^{25-27}$ and risky online experiences ${ }^{29,30}$. However, while belonging to offline primary groups was found to buffer the negative association between cybercrime victimization and SWB, this effect was not found in the case of online communities. Thus, our findings seem to show that the buffering effect does not apply to online and offline social belonging equivalently, at least in the case of cybercrime victimization. Parallel findings concerning the different function of social belonging in the offline and online environments have been reported in earlier studies ${ }^{29,30}$.

The divergence between online and offline belonging may be related to qualitative differences in communication offline and online ${ }^{31}$. According to social information processing theory, relationships built around computer-mediated communication require more time to reach equivalent 
levels of intimacy as is found in face-to-face relationships ${ }^{35}$. Also, users' own attitudes towards online communication might be the determining factor for intimacy in online relationships ${ }^{36}$. The online space is multifaceted, with any number of variations in social setting, interactional potential and investment, level of user visibility and social cohesion. Hence, online communication facilitates the establishing and maintaining of social relations that may in general be of lower intimacy than are those embedded in offline social networks ${ }^{37}$.

In general, young people's social relationships originated online last for a shorter time, are less intensive, and have fewer shared topics of interest than those formed offline ${ }^{38}$. The lack of an online buffering effect may, thus, be linked to belonging online being less emotionally meaningful to users despite online relations' importance in less intimate ways such as for gaining advice or the opportunity to share mutual experiences, for example. In line with this are Cole's and colleagues' findings where emotional support and frequency of interaction and social ties online were found to buffer the harmful effects of peer victimization ${ }^{28}$.

In addition, the difference between the two forms of belonging may be linked to the fact that people often interact with same social contacts in both offline and online contexts ${ }^{40}$ and, thus, online social belonging might appear as a mere extension of offline belonging. However, online social ties have been reported elsewhere as a distinct source of social support that is not reducible to offline social $\operatorname{ties}^{28}$. It is also possible that people prefer turning to their offline primary groups during stressful events and that online social belonging is an important form of social support primarily for those failing to receive it from the offline context.

\section{Limitations}

As our analyses were based on cross-sectional survey data, responses may contain a variety of biases in terms of assessing victimization severity and criminality classification. Firstly, the crosssectional data only allow for an analysis of the association, rather than causal inference. The hypothesized direction of the association between cybercrime victimization and SWB, as it was analyzed here, was based on previous research on crime victimization and well-being ${ }^{9-11}$. Secondly, the subjective measurement of sensitive issues such as victimization experiences has potential limitations concerning memory bias and the willingness to report such experiences, for example. However, the self-report approach to victimization research has been validated and utilized extensively in past studies ${ }^{41,42}$. Thirdly, it is possible that measuring the time passed since victimization experience and the severity of the crime would have allowed us to observe stronger effect sizes for crime victimization and to make different inferences on cybercrime victimization. These issues would undoubtedly be relevant for future research.

\section{Conclusion}

This study showed that cybercrime victimization experience in the past three years predicted lower SWB for adolescents and young adults. This was true in the case of cybercrime in general and its subtypes involving offensive cybercrime and cyberfraud. However, the negative association was buffered to a significant degree by social belonging to family, friends and school/workmates. Thus, even though cybercrimes and other harmful online experiences take place in technology-mediated environments, the buffering effect hypothesis and social support offered by offline primary groups remain relevant. Notably, social relations in the online community do not seem to have the same protective effect. 
As various peer support communities get more popular and accessible online it remains an important topic for examining the characteristics that separate these social ties from offline ones, at least when it comes to buffering of harmful online experiences. In addition, to facilitate the development of interventions for cybercrime victims, future research should further examine the exact mechanisms of social support that offline and online social networks do or could offer to protect young people from cybercrime victimization experiences. Although social belonging offline was identified here as a protective factor, the emotional, informational and instrumental support provided by social relations both offline and online for those victimized are potential sources of buffering as well.

\section{References}

1.Holt TJ, Bossler AM, Seigfried-Spellar KC. (2015) Cybercrime and digital forensics: An introduction. New York: Routledge.

2.Kirwan G, Power A. (2013) Cybercrime: The Psychology of Online Offenders. Cambridge: Cambridge University Press.

3.Leukfeldt ER, Yar M. Applying routine activity theory to cybercrime: A theoretical and empirical analysis. Deviant Behavior 2016; 37:263-280.

4. Näsi M, Oksanen A, Keipi T, Räsänen P. Cybercrime Victimization among Young People: A Multi-national Study. Journal of Scandinavian Studies in Criminology and Crime Prevention 2015; 16:203-210, DOI: 10.1080/14043858.2015.1046640.

5.Yar M. (2013) Cybercrime and society. London: Sage Publications.

6.McGuire M, Dowling S. (2013). Cyber crime: A review of the evidence. www.gov.uk/government/uploads/system/uploads/attachment data/file/246749/horr75summary.pdf (Accessed 09-Oct-2017).

7.Oksanen A, Keipi T. Young People as Victims of Crime on the Internet: A Population-based Study in Finland. Vulnerable Children \& Youth Studies 2013; 8:298-309. DOI: $10.1080 / 17450128.2012 .752119$

8.Williams ML. Guardians upon high: an application of routine activities theory to online identity theft in Europe at the country and individual level. British Journal of Criminology 2016; 56:21-48.

9.Davis RC, Friedman LN. (1985) The emotional aftermath of crime and violence. In: Figley C, ed. Trauma and its Wake: The Study and Treatment of Post-traumatic Stress Disorder. New York: Brunner/Mazel, pp. 90-112.

10.Tan SY, Haining R. Crime victimization and the implications for individual health and wellbeing: A Sheffield case study. Social Science \& Medicine 2016; 167:128-139.

11. Mahuteau S, Zhu R. Crime Victimisation and Subjective Well-Being: Panel Evidence From Australia. Health Economics 2016; 25:1448-1463. 
12.Diener E. Subjective well-being: The science of happiness and a proposal for a national index. American Psychologist 2000; 55:34-43.

13.Abdel-Khalek AM. Measuring happiness with a single-item scale. Social Behavior and Personality 2006; 34:139-150.

14.Dolan P, Peasgood T, White M. Do we really know what makes us happy? A review of the economic literature on the factors associated with subjective well-being. Journal of Economic Psychology 2008; 29:94-122.

15.Jorm A, Ryan SM. Cross-national and historical differences in subjective well-being. International Journal of Epidemiology 2014; 43:330-340.

16.Veenhoven R. (1984) Conditions of happiness. Dordrecht: Reidel.

17.Vakhitova ZI, Reynald DM, Townsley M. Toward the Adaptation of Routine Activity and Lifestyle Exposure Theories to Account for Cyber Abuse Victimization. Journal of Contemporary Criminal Justice 2016; 2: 169-188.

18.Näsi M, Räsänen P, Kaakinen M, Keipi T, Oksanen A. Do routine activities help predict young adults' online harassment: A multi-nation study. Criminology \& Criminal Justice 2016, online first. DOI: $10.1177 / 1748895816679866$.

19.Priebe G, Svedin CG. Online or offline victimisation and psychological well-being: a comparison of sexual-minority and heterosexual youth. European Child \& Adolescent Psychiatry 2012; 21:569-582.

20.Tennant JE, Demaray MK, Coyle S, Malecki CK. The dangers of the web: Cybervictimization, depression, and social support in college students. Computers in Human Behavior 2015; 50:348357.

21.Baumeister RF, Leary MR. The need to Belong: Desire for Interpersonal Attachments as a Fundamental Human Motivation. Psychological Bulletin 1995; 117:497-529.

22.Thoits P. Mechanisms Linking Social Ties and Support to Physical and Mental Health. Journal of Health and Social Behavior 2011; 52:145-161.

23.Cobb S. Social Support as a Moderator of Life Stress. Psychosomatic Medicine 1976; 38:300314.

24.Cohen S, Wills TA. Stress, social support, and the buffering hypothesis. Psychological Bulletin 1985; 98: 310-357.

25.Brooks M, Lowe M, Graham-Kevan N, Robinson S. Posttraumatic growth in students, crime survivors and trauma workers exposed to adversity. Personality and Individual Differences 2016; 98: 199-207.

26.Schultz D, Jaycox LH, Hickman, LJ, Setodji C, Kofner A, Harris R, Barnes D. The Relationship Between Protective Factors and Outcomes for Children Exposed to Violence. Violence and Victims 2013; 28: 697-714. 
27.Spillman S. Child abduction, Parrents' distress, and social support. Violence and Victims 2006; 21: 149-165.

28.Cole DA, Nick EA, Zelkowitz RL, Roeder KM, Spinelli T. Online social support for young people: Does it recapitulate in-person social support; can it help? Computers in Human Behavior 2017; 68: $456-464$.

29.Minkkinen J, Oksanen A, Näsi M, Keipi T, Kaakinen M, Keipi T, Räsänen P. Does social belonging to primary groups protect young people from the effects of pro-suicide sites? A comparative study of four countries. Crisis: The Journal of Crisis Intervention and Suicide Prevention 2016; 37: 31-41, DOI: 10.1027/0227-5910/a000356.

30.Turja T, Oksanen A, Kaakinen M, Sirola A, Kaltiala-Heino R, Räsänen P. Proeating disorder websites and subjective well-being: A four-country study on young people. International Journal of Eating Disorders 2016; online first: doi: 10.1002/eat.22589.

31.Spears R, Lea M, Postmes T. (2001) Social psychological theories of computer- mediated communication: Social gain or social pain? In: Robinson WP and Giles H, eds. The Handbook of Language and Social Psychology. Chichester: Wiley, pp 601-623.

32. Spears R, Postmes T, Lea M, Wolbert A. When Are Net Effects Gross Products? The Power of Influence and the Influence of Power in Computer-Mediated Communication. Journal of Social Issues 2002; 58: 91-107.

33.Keipi T, Oksanen A. Self-exploration, anonymity and risks in the online setting: Analysis of narratives by 14-18-year olds. Journal of Youth Studies 2014; 17:1097-1113.

34.Lehdonvirta V, Räsänen P. How do young people identify with online and offline peer groups? A comparison between UK, Spain and Japan. Journal of Youth Studies 2011; 14:91-108.

35.Walther JB. Interpersonal effects in computer-mediated interaction: A relational perspective. Communication Research 1992; 19: 52-90. doi:10.1177/009365092019001003.

36.Tanis M. Social Cues and Impression Formation in CMC. Journal of Communication 2003; 53: 676-693. doi:10.1093/joc/53.4.676.

37.Dibble JL, Drouin M, Aune KS, Boller RR. Simmering on the back burner: communication with and disclosure of relationship alternatives. Communication Quarterly 2015; 63: 329-344.

38.Mesch G, Talmud I. The Quality of Online and Offline Relationships: The Role of Multiplexity and Duration of Social Relationships. The Information Society 2006; 22: 137-148. doi: 10.1080/01972240600677805.

39.United Nations (2013). Comprehensive Study on Cybercrime. United Nations Office on Drugs and Crime: Vienna. www.unodc.org/documents/organizedcrime/UNODC_CCPCJ_EG.4_2013/CYBERCRIME_STUDY_210213.pdf (Accessed 09-Oct2017). 
40. Hampton KN, Goulet LS, Rainie L, Purcell K. Social networking sites and our lives: How people's trust, personal relationships, and civic and political involvement are connected to their use of social networking sites and other technologies. PEW Internet and American Life Project 2011. www.pewinternet.org/Reports/2011/Technology-and-social-networks.aspx (Accessed 09-Oct2017).

41.Cho B, Jackson Y. Self-reported and case file maltreatment: Relations to psychosocial outcomes for youth in foster care. Children and Youth Services Review 2017; 69:241-247.

42.Nedelec JL. A multi-level analysis of the effect of interviewer characteristics on survey respondents' reports of sensitive topics. Personality and Individual Differences 2017; 107:96-101. 
Table 1. Descriptive Statistics for Full Data and by Country

\begin{tabular}{|c|c|c|c|c|c|c|c|c|c|c|c|}
\hline \multirow[b]{2}{*}{ Continuous variables } & \multicolumn{3}{|c|}{ All countries } & \multicolumn{2}{|c|}{ USA } & \multicolumn{2}{|c|}{ UK } & \multicolumn{2}{|c|}{ Germany } & \multicolumn{2}{|c|}{ Finland } \\
\hline & range & mean & $\mathrm{SD}$ & mean & SD & mean & $\mathrm{SD}$ & mean & SD & mean & $\mathrm{SD}$ \\
\hline SWB & $2-20$ & 13.32 & 4.00 & 13.61 & 3.86 & 12.87 & 3.93 & 13.22 & 4.26 & 13.81 & 3.84 \\
\hline Social belonging offline & $3-15$ & 10.91 & 2.56 & 11.14 & 2.28 & 10.45 & 2.60 & 11.21 & 2.37 & 10.77 & 2.67 \\
\hline Social belonging online & $1-5$ & 2.42 & 1.17 & 2.50 & 1.20 & 2.26 & 1.20 & 2.32 & 1.08 & 2.74 & 1.16 \\
\hline Age & $15-30$ & 23.06 & 4.08 & 23.09 & 4.044 & 23.18 & 4.14 & 23.20 & 3.98 & 22.59 & 4.21 \\
\hline Scope of SNS activity & $0-19$ & 6.02 & 2.97 & 6.20 & 3.11 & 6.22 & 3.09 & 5.54 & 2.77 & 6.18 & 2.74 \\
\hline Categorical variables & coding & \multicolumn{2}{|c|}{ frequency $\% \quad(n)$} & \multicolumn{2}{|c|}{ frequency $\% \quad(n)$} & \multicolumn{2}{|c|}{ frequency $\% \quad(n)$} & \multicolumn{2}{|c|}{ frequency $\% \quad(n)$} & \multicolumn{2}{|c|}{ frequency $\% \quad(n)$} \\
\hline Female & dummy & 49.85 & 1777 & 50.34 & 520 & 49.05 & 490 & 50.10 & 490 & 49.91 & 277 \\
\hline Victim of cybercrime & dummy & 6.42 & 229 & 6.10 & 63 & 7.41 & 74 & 6.03 & 59 & 5.95 & 33 \\
\hline Victim of offensive cybercrime & dummy & 4.26 & 152 & 4.36 & 45 & 4.80 & 48 & 4.09 & 40 & 3.42 & 19 \\
\hline Defamation & dummy & 2.61 & 93 & 2.90 & 30 & 2.50 & 25 & 2.76 & 27 & 1.98 & 11 \\
\hline Ilegal threat & dummy & 2.19 & 78 & 1.65 & 17 & 2.70 & 27 & 2.35 & 23 & 1.98 & 11 \\
\hline Sexual harassment & dummy & 1.09 & 39 & 1.26 & 13 & 1.00 & 10 & 1.02 & 10 & 1.08 & 6 \\
\hline Victim of cyberfraud & dummy & 2.92 & 104 & 2.52 & 26 & 2.80 & 28 & 3.27 & 32 & 3.24 & 18 \\
\hline Identity theft & dummy & 1.49 & 53 & 1.65 & 17 & 1.60 & 16 & 1.12 & 11 & 1.62 & 9 \\
\hline Fraud & dummy & 1.77 & 63 & 1.16 & 12 & 1.60 & 16 & 2.35 & 23 & 2.16 & 12 \\
\hline $\mathrm{N}$ & & 35 & & 10 & & 99 & & 978 & & 55 & \\
\hline
\end{tabular}


Table 2. Hierarchical Linear Regression Models Predicting Subjective Well-Being with Cybercrime Victimization and Covariates

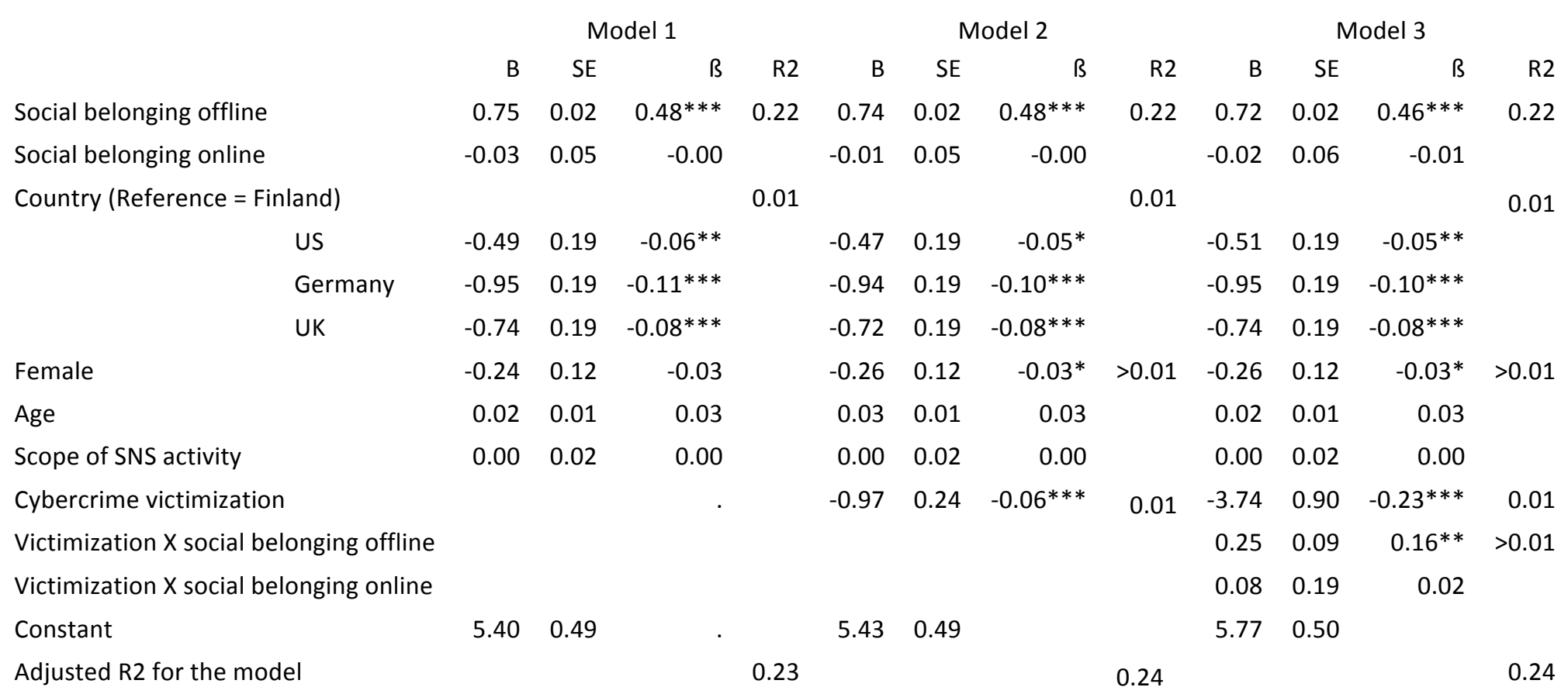

Note: ${ }^{* * *} \mathrm{p}<0.001,{ }^{* *} \mathrm{p}<0.01,{ }^{*} \mathrm{p}<0.05$ 
Table 3. Hierarchical Linear Regression Models Predicting Subjective Well-Being with Offensive Cybercrime Victimization and Covariates

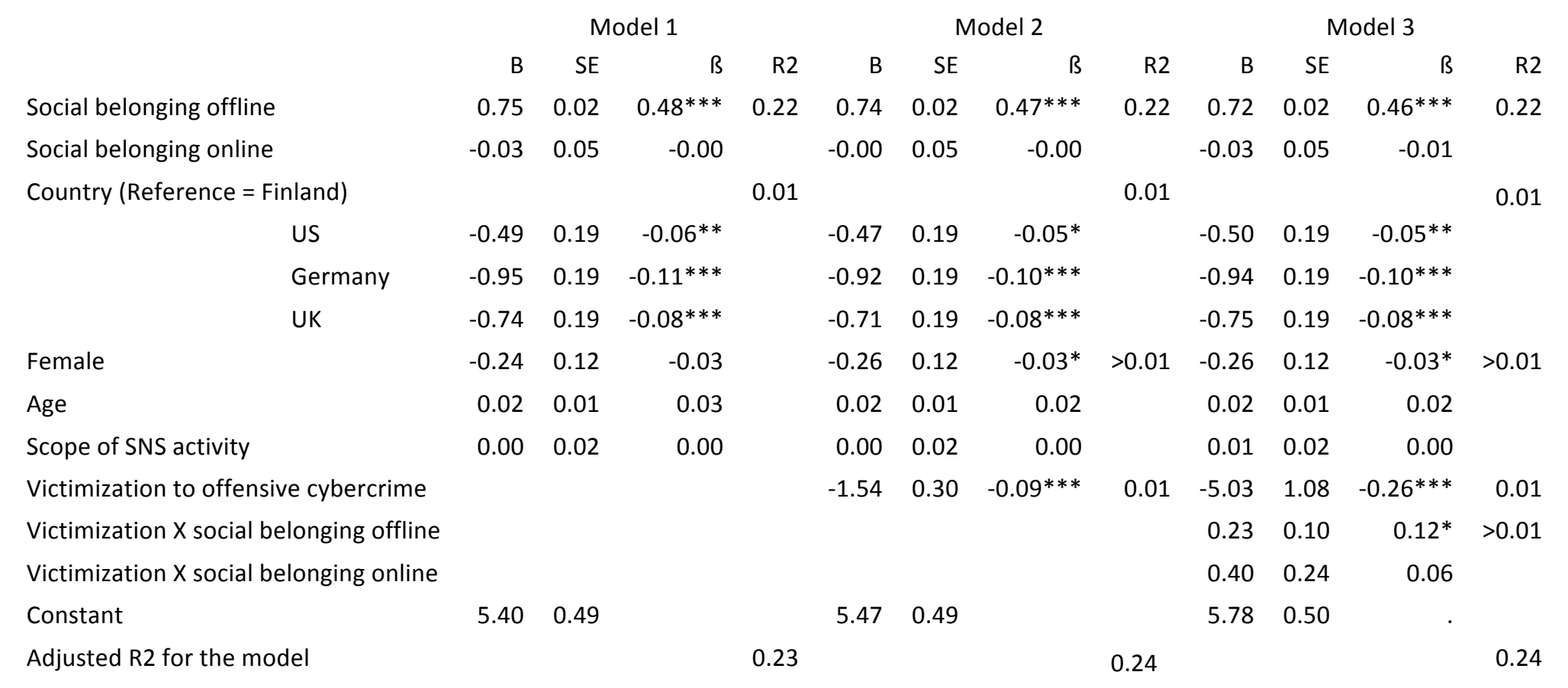

Note: ${ }^{* * *} \mathrm{p}<0.001,{ }^{* *} \mathrm{p}<0.01,{ }^{*} \mathrm{p}<0.05$ 
Table 4. Hierarchical Linear Regression Models Predicting Subjective Well-Being with Cyberfraud Victimization and Covariates

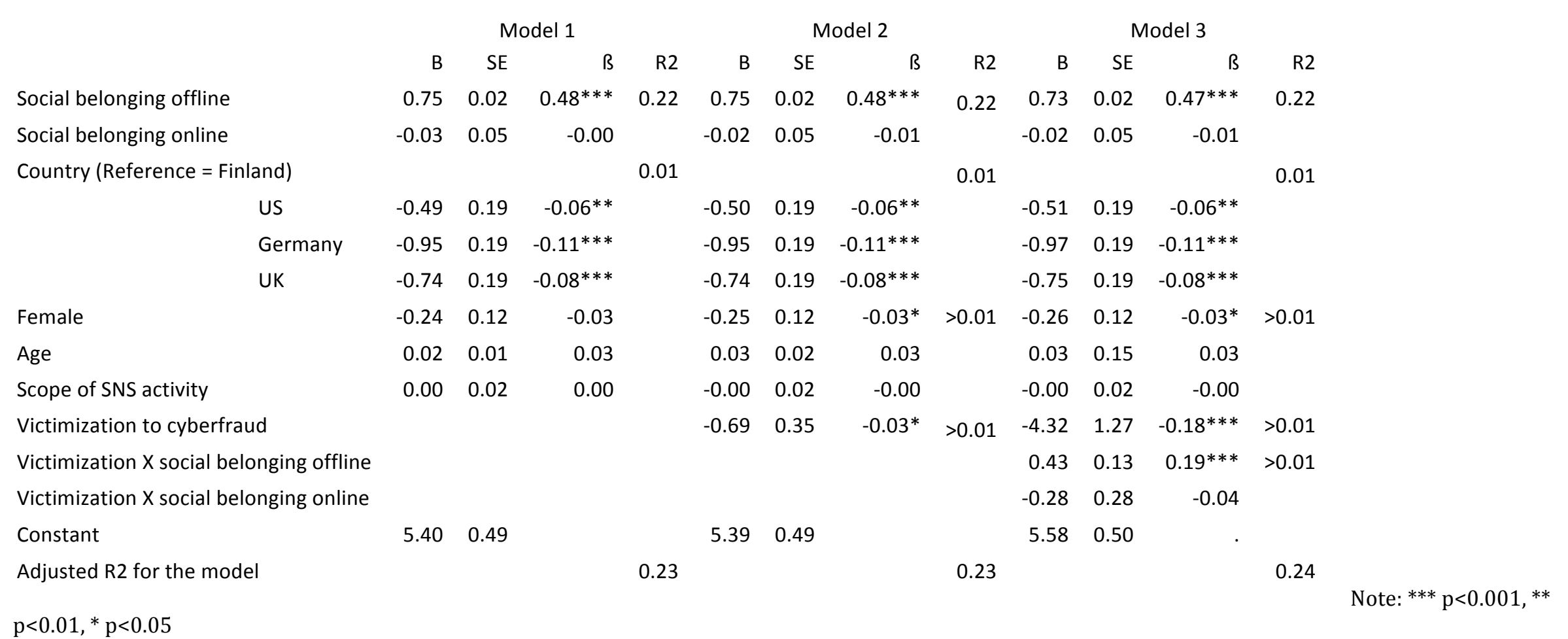


FIG. 1. Adjusted predictions of SWB with 95 percent CIs for the nonvictims and victims of cybercrime with low (1 SD below mean), average (mean), or high (1 SD above mean) social belonging offline. SWB, subjective well-being; CI, confidence interval; SD, standard deviation.

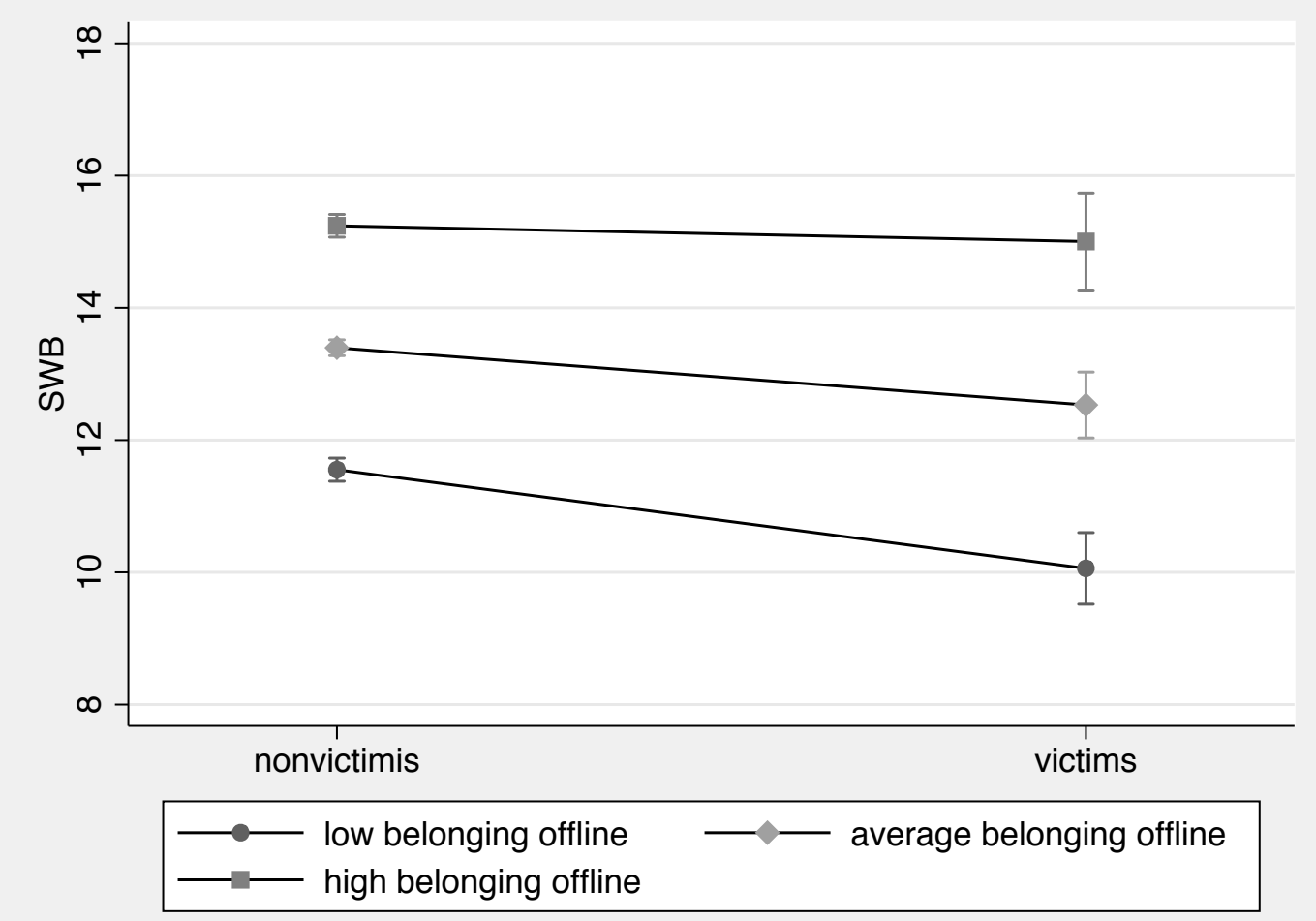


FIG. 2. Adjusted predictions of SWB with 95 percent CIs for the nonvictims and victims of offensive cybercrime with low (1 SD below mean), average (mean), or high (1 SD above mean) social belonging offline.

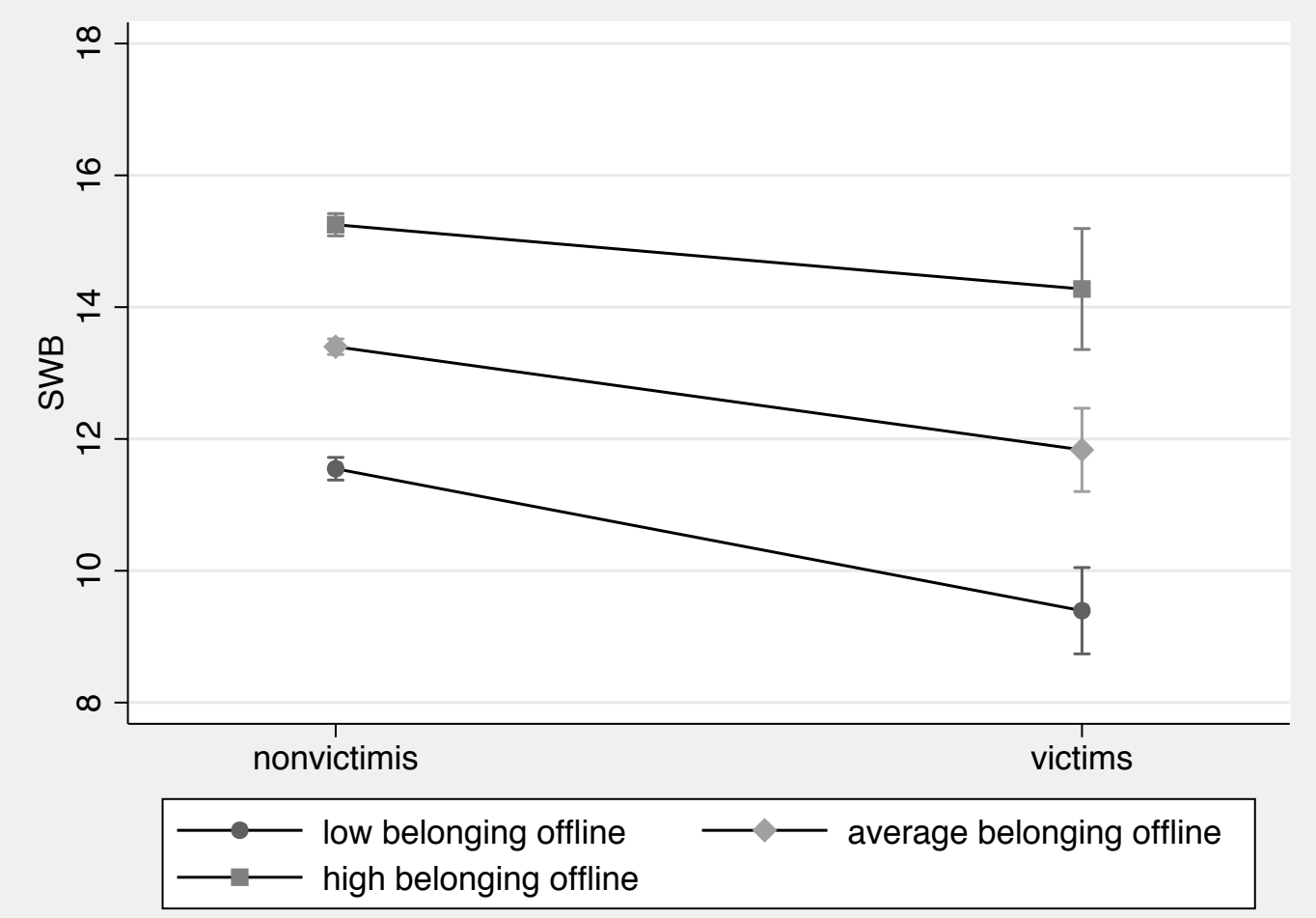


FIG. 3. Adjusted predictions of SWB with 95 percent CIs for the nonvictims and victims of cyberfraud with low (1 SD below mean), average (mean), or high (1 SD above mean) social belonging offline.

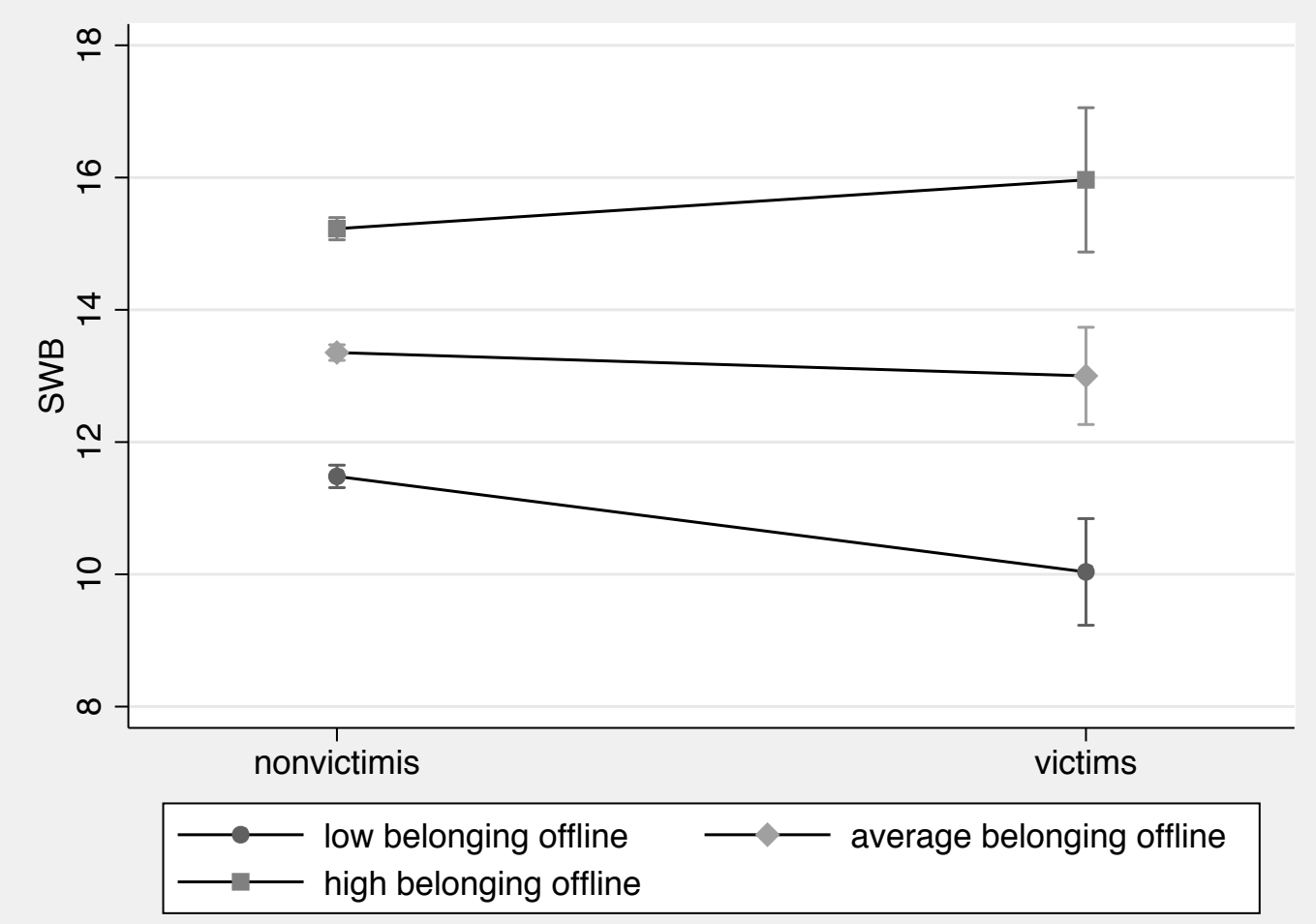

